Plus de $80 \%$ de la population adulte consulte au moins une fois par an un médecin installé. Les consultations médicales fournissent donc d'importantes occasions de parler de prévention avec les patients et de les motiver à changer de comportement. Cela vaut également pour le tabagisme, car il n'est jamais trop tard pour arrêter de fumer!

\title{
Patientes et patients fumeurs traités dans les cabinets médicaux
}

\author{
Auteure: Dr Michelle Dey ; coauteures: Verena El Fehrib, Anne-Katharina Burkhalter

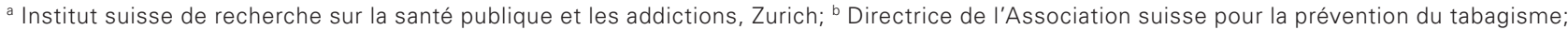 \\ "Responsable du projet "Vivre sans tabac»
}

En 2016, une enquête concernant les conseils en matière de sevrage tabagique a été réalisée auprès du corps médical. Il s'avère que dans les cabinets médicaux, la question de la désaccoutumance au tabac a encore gagné en importance par rapport aux années précédentes. En outre, les cours de formation continue ont un effet positif sur la propension des médecins à intervenir.

L’offre «Vivre sans tabac - conseil médical en désaccoutumance», proposée dans le cadre du Programme national d'arrêt du tabagisme, a pour but d'augmenter la qualité et la fréquence des conseils prodigués par les médecins aux patientes et patients fumeurs et, par voie de conséquence, du nombre de sevrages réussis en Suisse. La morbidité et la mortalité liées au tabagisme devraient s'en trouver réduites.

En 2016, le projet «Vivre sans tabac» a fait l'objet d'un sondage écrit pour la troisième fois (les évaluations

\section{Offres du Programme national d'arrêt du tabagisme}

\section{Concours pour arrêter de fumer 2017}

Un grand nombre de fumeuses et de fumeurs voudraient vraiment arrêter de fumer, mais ils appréhendent la première étape. Faire le pari d'arrêter de fumer pendant un mois avec d'autres personnes concernées est plus motivant que d'essayer tout seul. Le concours pour arrêter de fumer offre justement cette opportunité aux fumeuses et aux fumeurs. Toutes les personnes qui renonceront à fumer entre le $1^{\text {er }}$ et le 30 juin 2017 pourront prendre part au tirage au sort doté d'un prix principal de 5000 francs et de dix autres prix de 500 francs. Pour de nombreuses personnes, se passer de cigarettes pendant un mois marque le début d'une vie sans fumée. Vous trouverez toutes les informations concernant le concours pour arrêter de fumer sur www.concours-stop-tabac.ch.

Vous avez la possibilité de poser dans votre cabinet des affiches et de mettre à disposition des cartes de concours rédigées en allemand, français, italien, romanche, anglais, albanais, serbe/ croate/bosnien et turc. Vous pouvez les commander par téléphone au 03159910 20, par courriel à l'adresse info[at]at-schweiz.ch ou en ligne sur www.concours-stop-tabac.ch/shop

Le concours pour arrêter de fumer est organisé par le Programme national d'arrêt du tabagisme et soutenu entre autres par la Fédération des médecins suisses. Il est financé par le fonds de prévention du tabagisme. précédentes ayant été réalisées en 2002/2003 et 2008). Le questionnaire a été envoyé à un panel de médecins exerçant en cabinet privé (adresses valables $=3345$ ) Après trois envois (un envoi principal et deux rappels), le taux de réponse a atteint $36 \%$. Lorsque tous les professionnels n'ont pas répondu à la question, la somme des pourcentages indiqués n'atteint pas 100.

\section{Participant(e)s}

La plupart des participant(e)s étaient des spécialistes en médecine générale et médecine interne $(56,9 \%)$, suivis des médecins spécialisés dans les domaines de la gynécologie (18\%), de la pneumologie $(8,6 \%)$ et de la pédiatrie (7,2\%). 7,9\% des répondants ont été classés dans la catégorie "Autre(s)»; il s'agissait en majorité de médecins spécialisés dans plusieurs disciplines. La majeure partie des personnes interrogées étaient établies en Suisse alémanique (55,3\%), de sexe masculin $(57,5 \%)$ et dans la fleur de l'âge. La majorité des participant(e)s n'avaient jamais fumé $(64,8 \%)$ ou avaient cessé leur consommation (26\%); suivaient les fumeurs occasionnels $(5,2 \%)$, tandis que les fumeurs quotidiens fermaient la marche (3\%). La prévalence des fumeurs a donc diminué au fil des ans. 


\section{Appréciation et recommandation d'arrêter de fumer}

La plupart des médecins $(85,9 \%)$ ont indiqué qu'ils interrogeaient leurs patientes et patients sur leur comportement en matière de tabagisme. Cette proportion était considérablement plus élevée chez les pneumologues $(98,1 \%)$ et les gynécologues (95\%). Par ailleurs, le nombre de médecins qui demandent à l'ensemble de leurs patient(e)s s'ils sont fumeurs a légèrement augmenté au fil du temps. 9,3\% des personnes interrogées se contentent de poser la question uniquement aux patient(e)s présentant un tableau clinique typique et $2,6 \%$ n'interrogent jamais leurs patient(e)s à ce sujet (très souvent chez les pédiatres).

La plupart des participant(e)s $(80,8 \%)$ indiquent toujours le statut de fumeur des patient(e)s dans leur dossier. 16,6\% d'entre eux le font occasionnellement, tandis que $2 \%$ ne documentent jamais ce statut.

Au total, 86,9\% des sondé(e)s recommandent à tous les fumeurs de cesser leur consommation. Le pourcentage est particulièrement élevé parmi les pneumologues (98,1\%). 39,2\% des participant(e)s qui conseillent d'arrêter de fumer recommandent à leurs patient(e)s de se faire aider. Chez les pneumologues, le pourcentage correspondant atteint 59,6\%. La moitié des médecins $(50,4 \%)$ ne formulent une recommandation de ce genre que lorsque la patiente ou le patient manifeste de l'intérêt, et 9,4\% ne font jamais de recommandations à ce sujet. Les médecins qui conseillent d'avoir recours à une aide pour arrêter de fumer recommandent surtout les substituts nicotiniques (66,7\%), prescrivent de la varénicline $(64,4 \%)$, proposent à leurs patient(e)s un accompagnement personnel durant le sevrage $(60,2 \%)$, recommandent des cours sur l'arrêt du tabagisme $(40,6 \%)$ et orientent les patient(e)s vers des consultations spécialisées (30\%).

Pour expliquer qu'ils renoncent à offrir un soutien lors du sevrage ou à faire des recommandations, les médecins invoquent le plus souvent une formation insuffisante dans ce domaine (39\%), un manque d'intérêt de la part du fumeur (37,5\%) et le manque de temps $(32,8 \%)$.

\section{Formation et formation continue en matière de désaccoutumance au tabac}

Un quart des personnes interrogées (25,6\%) déclarent avoir suivi des cours sur le thème du tabagisme durant leur formation de médecin. Ce pourcentage est plus élevé parmi les plus jeunes $(36,4 \%$ chez les moins de 39 ans). 33,8\% des médecins ont déjà suivi des cours de formation continue sur les conseils médicaux en dés-

\section{VIVRE SANS TABAC - conseil médical}

\section{en désaccoutumance}

Outre la documentation de base destinée au corps médical et les brochures élaborées à l'intention des patientes et des patients, le projet propose surtout des cours de formation continue gratuits dans toute la Suisse. Pour chaque cours, la SSMIG attribue des crédits pour la formation continue essentielle MIG.

- Genève, 8 juin et 22 juin 2017, en deux parties, de 8 h30 à 12h30, HUG

- Lausanne, 7 septembre 2017, de 14h à 17h, PMU; 29 novembre 2017 , de $13 \mathrm{~h} 30$ à $15 \mathrm{~h}, \mathrm{PMU}$

- Lugano, 10 giugno 2017, dalle 9:00 alle 16:30, formazione per medici e paramedici, Lega polmonare ticinese

- Mendrisio, 25 novembre 2017, dalle 9:00 alle 16:30, formazione per medici e paramedici, Ospedale Regionale

ASTA (Apprentissages spécifiques en tabacologie pour médecins assistants)

Nouveauté: désormais de nombreuses cliniques $A$ proposent des cours succincts spécialement prévus pour les médecins en cours de formation postgraduée en médecine générale et médecine interne. Contact et inscription par courriel à: annekburkhalter[at]freivon tabak.ch

accoutumance. Cette proportion est plus importante parmi les pneumologues (64,8\%). Au cours des années, la participation à des cours a augmenté. Les médecins qui ont suivi un cours de formation continue ont davantage tendance que les autres à interroger leurs patient(e)s quant à leur statut de fumeur et à le documenter, à leur recommander d'arrêter de fumer et de se faire aider dans cette démarche, et à leur apporter du soutien durant le sevrage. Les informations régulières sur la désaccoutumance au tabac semblent elles aussi avoir un effet positif sur les pratiques médicales.

\section{Conclusion}

En résumé, on constate plusieurs améliorations par rapport aux sondages précédents, par exemple l'augmentation du nombre de médecins qui interrogent toutes les patientes et tous les patients au sujet de leur consommation de tabac ou encore celle du nombre de professionnels ayant déjà suivi un cours dans ce domaine. Pour terminer, il a été possible de mettre en évidence des variables que l'on pouvait influencer (par ex. la fréquentation de cours) et qui ont ensuite un effet positif sur la manière dont les médecins appréhendent les patientes et patients fumeurs. Il reste toutefois beaucoup à faire. Il faudrait notamment réduire encore davantage l'absence de formation dans le domaine du sevrage tabagique, un motif que les médecins invoquent encore pour justifier qu'ils ne fournissent pas de soutien lors du sevrage, ou qu'ils ne le font que rarement. 